Kardiologe 2021 · 15:175

https://doi.org/10.1007/s12181-021-00449-w

Angenommen: 18. Januar 2021

Online publiziert: 9. Februar 2021

(c) Springer Medizin Verlag GmbH, ein Teil von Springer Nature 2021

\section{Originalpublikation}

Zhang JJ et al (2020) Multicentre, randomized comparison of two-stent and provisional stenting techniques in patients with complex coronary bifurcation lesions: the DEFINITION II trial. European Heart Journal 41:2523-2536. https://doi.org/10.1093/eurheartj/ehaa543

Bei komplexen Bifurkationsläsionen mit Beteiligung von Hauptgefäß und „Seitenast“" stellt sich nicht nur im Bereich des linkskoronaren Hauptstammes die Frage, ob eine Versorgung mit einer 2-StentStrategie günstiger ist als eine 1-StentStrategie (sog. „provisional stenting“).

\section{Zusammenfassung der Studie}

Die DEFINITION II-Studie ist dieser Frage nachgegangen und hat 660 Patienten mit komplexer Koronarbifurkation, die sich einer PCI unterzogen haben, auf "provisional stenting“ (1-Stent-Strategie) vs. geplante 2-Stent-Strategie randomisiert. Komplexe Koronarbifurkation (nur Medina 1,1,1 oder 0,1,1 Stenosen wurden eingeschlossen) wurde definiert als Stenose des Hauptgefäßes plus Seitenastläsion $\geq 10 \mathrm{~mm}$ und -stenose $\geq 70 \%$ bei distalen Hauptstammstenosen bzw. Seitenastläsion $\geq 10 \mathrm{~mm}$ und -stenose $\geq 90 \%$ bei allen übrigen $\mathrm{Bi}$ furkationen zuzüglich von mindestens 2 der folgenden Kriterien: mindestens mittelgradige Verkalkungen, multiple Läsionen, Bifurkationswinkel $<45^{\circ}$ oder $>70^{\circ}$, Durchmesser des Hauptgefäßes $<2,5 \mathrm{~mm}$, Thrombus im Bereich der Läsionen oder Läsionslänge im Hauptgefäß $>25 \mathrm{~mm}$. Für die geplante 2-Stent-Strategie wurde explizit die DK Crush(double

Jan-Malte Sinning

Innere Medizin III - Kardiologie, St. Vinzenz-Hospital, Köln, Deutschland

\title{
Komplexe Bifurkationsläsionen - Zwei Stents sind doch besser als einer!
}

kissing crush)- bzw. Culotte-StentingTechnik empfohlen.

Die 660 Patienten wurden auf beide Gruppen aufgeteilt und in der Zeit von Dezember 2015 bis November 2018 in 49 internationalen Zentren randomisiert und 12 Monate nachbeobachtet. In beiden Gruppen erhielten jeweils mehr als $50 \%$ ein angiographisches Follow-up. Der primäre Endpunkt war eine Kombination von „target lesion failure“ (TLF) nach 1 Jahr Follow-up inklusive kardialer Tod, „target vessel myocardial infarction“ (TVMI) und klinisch bedingter „target lesion revascularization" (TLR).

Nach 1 Jahr Follow-up trat der primäre Endpunkt TLF bei 37 (11,4\%) der Patienten in der "provisional stenting“ bzw. $20(6,1 \%)$ in der 2-Stent-Strategie-Gruppe auf. In dieser Gruppe war bei 77,8\% der Patienten ein „DK crush stenting" durchgeführt worden. Hiermit konnte das Risiko für den primären Endpunkt um $48 \%$ reduziert werden (Hazard Ratio [HR] 0,52, 95\%-Konfidenzintervall [KI] 0,30-0,90; $p=0,019$ ). Dies war v. a. bedingt durch TVMI $(7,1 \%$ vs. 3,0\%; $p=0,025)$ und klinisch bedingte $\operatorname{TLR}(5,5 \%$ vs. $2,4 \%, p=0,049)$ in der Provisional-stenting-Gruppe. Der Sicherheitsendpunkt Stentthromboserate sowie die kardiale Todesrate unterschieden sich nach 1 Jahr nicht zwischen den beiden Gruppen.

\section{Kommentar des Autors}

Die DEFINITION II-Studie hat große Relevanz für die Klinik: Bei komplexen Bifurkationsläsionen war in dieser Studie eine geplante 2-Stent-Strategie überlegen. Insbesondere DK Crush-
Stenting hat sich in mehreren Studien (DKCRUSH-Studien) als die beste 2-Stent-Strategie mit der niedrigsten Reinterventionsrate gezeigt. Ein Nachteil der Studie ist allerdings, dass nur bei $27,7 \%$ intravaskulärer Ultraschall zur Optimierung des Interventionsergebnisses benutzt wurde. Wenn - insbesondere bei Hauptstammläsionen - eine intravaskuläre Bildgebung routinemäßig zum Einsatz gekommen wäre, wäre das Ergebnis vielleicht noch günstiger gewesen. Eine randomisierte Studie zu dieser Thematik, insbesondere im Vergleich zur Bypasschirurgie, wäre sicherlich gerade bei Patienten mit Hauptstammläsionen sinnvoll. Das weitere Follow-up der Studie bleibt abzuwarten.

Zusammenfassend zeigt die DEFINITION II-Studie jedoch, dass eine 2-StentStrategie, wenn sie geplant durchgeführt wird, bei komplexeren Bifurkationsläsionen einen Vorteil haben kann.

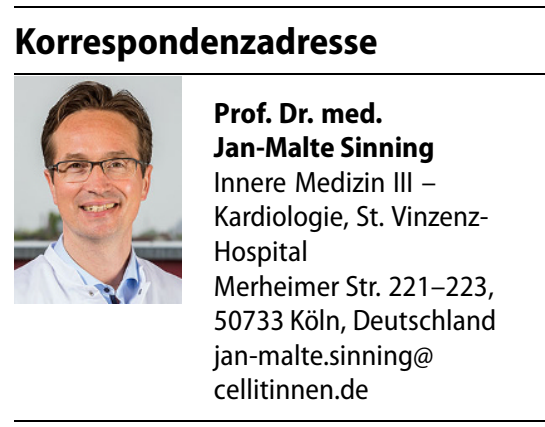

Interessenkonflikt. J.-M. Sinning: Forschungsförderung: Boston Scientific, Edwards Lifesciences und Medtronic. Beratertätigkeit: Abbott, Abiomed, Boehringer Ingelheim, Boston Scientific und Medtronic. Vortragshonorare und Reisekosten: Abbott, Abiomed, AstraZeneca, Bayer, Boehringer Ingelheim, Boston Scientific, Bristol-Myers Squibb, Edwards Lifesciences, Medtronic und Novartis. 